\title{
Cancer-Specific Mortality among Korean Men with Localized or Locally Advanced Prostate Cancer Treated with Radical Prostatectomy Versus Radiotherapy: A Multi-center Study Using Propensity Scoring and Competing Risk Regression Analyses
}

\section{Kyo Chul Koo, PhD ${ }^{1}$ \\ Jin Seon Cho, PhD² \\ Woo Jin Bang, MMS 2 \\ Seung Hwan Lee, $\mathrm{PhD}^{3}$ \\ Sung Yong Cho, $\mathrm{PhD}^{4}$ \\ Sun II Kim, $P h D^{5}$ \\ Se Joong Kim, PhD 5 \\ Koon Ho Rha, PhD ${ }^{3}$ \\ Sung Joon Hong, $\mathrm{PhD}^{3}$ \\ Byung Ha Chung, $\mathrm{PhD}^{1}$}

\begin{abstract}
${ }^{1}$ Department of Urology, Gangnam Severance Hospital, Yonsei University College of Medicine, Seoul, ${ }^{2}$ Department of Urology, Hallym University College of Medicine, Chuncheon, ${ }^{3}$ Department of Urology, Severance Hospital, Yonsei University College of Medicine, Seoul, ${ }^{4}$ Department of Urology, Inje University College of Medicine, Busan, ${ }^{5}$ Department of Urology, Ajou University School of Medicine, Suwon, Korea
\end{abstract}

\section{Purpose}

Studies comparing radical prostatectomy (RP) outcomes with those of radiotherapy with or without androgen deprivation therapy (RT \pm ADT) for prostate cancer (PCa) have yielded conflicting results. Therefore, we used propensity score-matched analysis and competing risk regression analysis to compare cancer-specific mortality (CSM) and other-cause mortality (OCM) between these two treatments.

\section{Materials and Methods}

The multi-center, Severance Urological Oncology Group registry was utilized to identify 3,028 patients with clinically localized or locally advanced PCa treated by RP $(n=2,521)$ or RT \pm ADT $(n=507)$ between 2000 and 2016. RT \pm ADT cases $(n=339)$ were matched with an equal number of RP cases by propensity scoring based on age, preoperative prostate-specific antigen, clinical tumor stage, biopsy Gleason score, and Charlson Comorbidity Index (CCl). CSM and OCM were co-primary endpoints.

\section{Results}

Median follow-up was 65.0 months. Five-year overall survival rates for patients treated with RP and RT \pm ADT were $94.7 \%$ and $92.0 \%$, respectively ( $p=0.105)$. Cumulative incidence estimates revealed comparable CSM rates following both treatments within all National Comprehensive Cancer Network risk groups. Gleason score $\geq 8$ was associated with higher risk of CSM ( $p=0.009)$. OCM rates were comparable between both groups in the low- and intermediate-risk categories $(p=0.354$ and $p=0.643$, respectively). For high-risk patients, RT \pm ADT resulted in higher OCM rates than RP $(p=0.011)$. Predictors of OCM were age $\geq 75$ years $(p=0.002)$ and $\mathrm{CCl} \geq 2(p<0.001)$.

\section{Conclusion}

$\mathrm{RP}$ and RT \pm ADT provide comparable CSM outcomes in patients with localized or locally advanced PCa. The risk of OCM may be higher for older high-risk patients with significant comorbidities.
Correspondence: Byung Ha Chung, MD, PhD Department of Urology, Gangnam Severance Hospital, Yonsei University College of Medicine, 211 Eonju-ro, Gangnam-gu, Seoul 06273, Korea Tel: 82-2-2019-3470

Fax: 82-2-3462-8887

E-mail: chung646@yuhs.ac

Received January 2, 2017

Accepted March 3, 2017

Published Online March 8, 2017

${ }^{*}$ Kyo Chul Koo and Jin Seon Cho contributed equally to this work.
Key words

Prostatic neoplasms, Prostatectomy, Radiotherapy,

Treatment outcome 


\section{Introduction}

The management of clinically localized and locally advanced prostate cancer ( $\mathrm{PCa}$ ) is controversial. Contemporary guidelines recommend that treatment decisions should be made on the basis of tumor features, baseline prostatespecific antigen (PSA) levels, patient age, comorbidity, life expectancy, and quality of life $[1,2]$. In general, radical prostatectomy $(\mathrm{RP})$ and radiation therapy with or without androgen deprivation therapy ( $\mathrm{RT} \pm \mathrm{ADT})$ are viable treatment options for patients with a life expectancy of more than 10 years [2].

Several studies have investigated the oncological outcomes of RP and RT \pm ADT in order to identify the population that would most benefit from a specific treatment and to determine which treatment is superior in terms of improving the length or quality of life [3-8]. However, most of these studies were retrospective in nature, and were limited by methodological biases arising from differences in pretreatment patient and cancer risk features between treatment cohorts $[9,10]$. Therefore, their results were inconclusive and yielded only weak evidence regarding which treatment was superior in terms of oncological outcome.

A randomized controlled trial is the ideal approach for comparing competing treatment modalities [11,12]. However, treatment options for PCa vary and decisions are largely based on patient preference and physicians' discretion. Compared to candidates for RP, patients who are offered RT generally tend to be older, have higher comorbidity scores, and have cancer-related risk features that are more aggressive, making a randomized trial impractical $[9,13]$. For instance, two large United States randomized trials comparing RP and RT were closed early because of poor accrual [14]. A feasible alternative is a propensity-score matched analysis, in which the possibility of patient selection bias can be minimized by adjusting for multiple preoperative confounders that may affect survival outcome, such as patient age, disease risk, and comorbidity [15]. The long lifespan of patients with subclinical PCa presents another hurdle in addressing the effect of a specific treatment on clinically relevant endpoints that truly represent the effect of a specific treatment, such as cancer-specific mortality (CSM) and other-cause mortality (OCM). Herein, a competing risks regression analysis can be used to better understand the magnitude and timeline in which a specific treatment might be expected to improve these survival endpoints.

To address these issues, we performed a propensity scorematched analysis followed by competing risk regression analyses to compare CSM and OCM outcomes between RP and $\mathrm{RT} \pm \mathrm{ADT}$ in a multi-center cohort of Korean patients with localized or locally advanced PCa. We further stratified our analyses according to the National Comprehensive Cancer Network (NCCN) PCa risk category so that our results could aid clinical decision making.

\section{Materials and Methods}

\section{Study population and data collection}

A total of 3,082 consecutive Korean patients with localized or locally advanced PCa treated with curative intent were selected from the multi-center, Severance Urological Oncology Group PCa registry. Of these, 2,521 patients (81.8\%) underwent RP and 561 patients (18.2\%) received RT \pm ADT between 2000 and 2016. The decision to use RP or RT \pm ADT for treatment was based on surgeons' discretion and on patients' preference. Ninety-four patients $(3.0 \%)$ with incomplete clinical information, 201 patients $(6.5 \%)$ who were lost to follow-up, and 107 patients (3.5\%) for both reasons were excluded from propensity-score calculation. This study was approved by the institutional ethics committee after review of the protocol and procedures employed (2014-0091-004).

\section{Radical prostatectomy}

$\mathrm{RP}$ was recommended for patients who either desired surgical treatment or were determined to be reasonable surgical candidates because of otherwise favorable clinical characteristics. Surgery was performed by the retropubic or robotic approach, with the extent of pelvic lymph node dissection being based upon the risk category of the patient.

\section{Radiation therapy}

Radio-oncologists of each participating institution confirmed that conventional or hypo-fractionated external beam RT was delivered to the prostate with pre-defined margins according to the guidelines of the European Organization for Research and Treatment of Cancer [16]. At Gangnam and Shinchon Severance Hospitals, RT consisted of 3D conformal radiation therapy (3DCRT) from 2000 to 2007 and intensity modulated external beam RT (IMRT) from 2007 to 2016. The median RT dose at Severance Hospitals was 7,000 cGy (interquartile range [IQR], 7,000 to 7,000 cGy). At Hallym University College Hospital, RT consisted of 3DCRT from 2000 to 2001 and IMRT from 2001 to 2016. The median RT dose at Hallym University College Hospital was 8,000 cGy (IQR, 8,000 to 8,000 cGy). At Ajou University Hospital, RT consisted of 3DCRT from 2000 to 2009 and IMRT from 2009 to 2016. The median RT dose at Ajou University Hospital was 
Table 1. Clinicopathological characteristics of patients, by initial treatment modality

\begin{tabular}{|c|c|c|c|}
\hline Characteristic & $R P(n=339)$ & $\mathrm{RT} \pm$ ADT $(\mathrm{n}=339)$ & p-value \\
\hline \multicolumn{4}{|l|}{ Propensity matched variable } \\
\hline Age (yr) & $70.0(66.0-73.0)$ & $70.1(66.0-74.0)$ & 0.629 \\
\hline PSA (ng/mL) & $10.4(6.7-20.7)$ & $10.7(7.0-21.5)$ & 0.814 \\
\hline \multicolumn{4}{|l|}{ Biopsy Gleason score (\%) } \\
\hline$\leq 6$ & $78(23.0)$ & $78(23.0)$ & $>0.99$ \\
\hline 7 & $133(39.2)$ & $133(39.2)$ & \\
\hline $8-9$ & $128(37.8)$ & $128(37.8)$ & \\
\hline \multicolumn{4}{|l|}{ Clinical T stage $(\%)$} \\
\hline cT1 & $79(23.3)$ & $79(23.3)$ & $>0.99$ \\
\hline cT2 & $140(41.3)$ & $140(41.3)$ & \\
\hline cT3 & $99(29.2)$ & $99(29.2)$ & \\
\hline cT4 & $21(6.2)$ & $21(6.2)$ & \\
\hline \multicolumn{4}{|l|}{$\mathrm{CCI}$} \\
\hline 0 & $224(66.1)$ & $224(66.1)$ & $>0.99$ \\
\hline 1 & $82(24.2)$ & $82(24.2)$ & \\
\hline$\geq 2$ & $33(9.7)$ & $33(9.7)$ & \\
\hline \multicolumn{4}{|l|}{ Unmatched variable } \\
\hline \multicolumn{4}{|l|}{ Clinical N stage $(\%)$} \\
\hline N0 & $322(95.0)$ & $322(95.0)$ & $>0.99$ \\
\hline N1 & $17(5.0)$ & $17(5.0)$ & \\
\hline \multicolumn{4}{|l|}{ Clinical M stage (\%) } \\
\hline M0 & $339(100)$ & $339(100)$ & NS \\
\hline M1 & 0 & 0 & \\
\hline Body mass index $\left(\mathrm{kg} / \mathrm{m}^{2}\right)$ & $23.7(22.3-25.5)$ & $23.6(21.7-25.6)$ & 0.948 \\
\hline \multicolumn{4}{|l|}{ NCCN risk criteria } \\
\hline Low & $23(6.8)$ & $22(6.5)$ & 0.985 \\
\hline Intermediate & $107(31.9)$ & $108(32.2)$ & \\
\hline High & $209(61.3)$ & $209(61.3)$ & \\
\hline Total follow-up period (mo) & $69.0(42.7-94.0)$ & $60.5(39.0-98.0)$ & 0.789 \\
\hline
\end{tabular}

Values are presented as median (interquartile range) and number (\%). RP, radical prostatectomy; RT \pm ADT, radiotherapy with or without androgen deprivation therapy; PSA, prostate-specific antigen; CCI, Charlson comorbidity index; NS, not significant; NCCN, National Comprehensive Cancer Network.

7,400 cGy (IQR, 7,010 to 7,400 cGy). The complete conversion from 3DCRT to IMRT at all participating institutions took place in 2011 along with the National Health Insurance Service reimbursement coverage. In overall, 216 (63.7\%) and 123 $(36.3 \%)$ patients received of 3DCRT and IMRT, respectively. The median total radiation dose was $70 \mathrm{~Gy}$ (IQR, 70 to $74 \mathrm{~Gy}$ ) in 33.5 fractions (IQR, 28 to 37 fractions), in which 295 patients $(87.0 \%)$ received greater than 7,600 cGy.

Pelvic lymph nodes were included if the patient had regional lymphadenopathies. Neoadjuvant, concomitant, and/or adjuvant ADT was performed in 13/22 (59.1\%), $69 / 108(63.9 \%)$, and 186/209 (88.9\%) of low-, intermediate-, and high-risk patients, respectively.

\section{Study endpoints}

CSM and OCM were the co-primary endpoints. For all patients, the status of survival and cause of death were investigated using institutional electronic medical records, the National Cancer Registry Database, or the Social Security Death Index. Death was attributed to PCa if evidence of progressive metastatic castration-resistant PCa (CRPC) was present, PCa was listed on the death certificate as the cause of death, or if the patient died of complications of PCa treatment. Secondary endpoints were biochemical recurrence-free survival (BCRFS), adjuvant therapy following recurrence, metastasis-free survival, and progression to CRPC-free survival. All patients received standard care according to contemporary guidelines until death or last follow-up. 
Table 2. Causes of death and 5-year survival rates stratified by risk category and initial treatment modality

\begin{tabular}{|c|c|c|c|}
\hline Variable & $R P(n=339)$ & $\mathrm{RT} \pm \mathrm{ADT}(\mathrm{n}=339)$ & p-value \\
\hline Deaths, n (\%) & $29(8.6)$ & $46(13.6)$ & 0.038 \\
\hline $\mathrm{PCa}$ & $6(1.7)$ & $4(1.2)$ & 0.530 \\
\hline Other cause & $23(6.8)$ & $42(12.4)$ & 0.013 \\
\hline Second primary malignancy & $7(2.1)$ & $13(3.8)$ & 0.758 \\
\hline Cardiopulmonary disease & $3(0.9)$ & $7(2.1)$ & 0.645 \\
\hline Unknown & $13(3.8)$ & $22(6.5)$ & 0.532 \\
\hline \multicolumn{4}{|l|}{ 5-Year survival rate $(\%)$} \\
\hline Cancer-specific & 98.8 & 99.5 & 0.576 \\
\hline Low risk & 100 & 100 & NS \\
\hline Intermediate risk & 100 & 100 & 0.994 \\
\hline High risk & 98 & 99.2 & 0.399 \\
\hline Other-cause & 95.3 & 93.0 & 0.051 \\
\hline Low risk & 100 & 100 & NS \\
\hline Intermediate risk & 94.2 & 90.5 & 0.863 \\
\hline High risk & 95.2 & 92.9 & 0.011 \\
\hline Overall & 94.7 & 92.0 & 0.105 \\
\hline Low risk & 100 & 100 & NS \\
\hline Intermediate risk & 94.2 & 90.5 & 0.871 \\
\hline High risk & 93.3 & 92.1 & 0.047 \\
\hline
\end{tabular}

$\mathrm{RP}$, radical prostatectomy; $\mathrm{RT} \pm \mathrm{ADT}$, radiotherapy with or without androgen deprivation therapy; $\mathrm{PCa}$, prostate cancer; NS, not significant.

Table 3. Oncological outcomes, by initial treatment modality

\begin{tabular}{|c|c|c|c|}
\hline Variable & $\mathbf{R P}(\mathrm{n}=339)$ & $\mathrm{RT} \pm$ ADT $(\mathrm{n}=339)$ & p-value \\
\hline \multicolumn{4}{|l|}{ BCR } \\
\hline No. $(\%)$ & 108 (31.9) & $57(16.8)$ & $<0.001$ \\
\hline Time to BCR (mo) & $17.0(7.0-34.5)$ & $40.0(15.0-57.5)$ & $<0.001$ \\
\hline 5-Year BCRFS (\%) & 3.7 & 22.8 & $<0.001$ \\
\hline \multicolumn{4}{|l|}{ Adjuvant therapy following BCR } \\
\hline Observation & $16(4.7)$ & $11(3.2)$ & 0.103 \\
\hline Salvage RT & $18(5.3)$ & $2(0.6)$ & \\
\hline ADT & $44(13.0)$ & $40(11.8)$ & \\
\hline Salvage RT plus ADT & $30(8.8)$ & $4(1.2)$ & \\
\hline \multicolumn{4}{|l|}{ CRPC $(\%)$} \\
\hline No. $(\%)$ & $16(4.7)$ & $14(4.1)$ & 0.721 \\
\hline Time to CRPC progression (mo) & $35.5(22.5-56.8)$ & $60.5(52.3-70.3)$ & 0.013 \\
\hline CRPC progression-free survival (\%) & 18.8 & 42.9 & 0.071 \\
\hline Chemotherapy & $7(2.1)$ & $9(2.7)$ & 0.603 \\
\hline \multicolumn{4}{|l|}{ Metastasis } \\
\hline No. $(\%)$ & $12(3.5)$ & $12(3.5)$ & $>0.99$ \\
\hline Time to metastasis (mo) & $45.0(26.0-71.3)$ & $54.5(24.5-68.0)$ & 0.839 \\
\hline Metastasis-free survival rate $(\%)$ & 33.3 & 41.7 & 0.778 \\
\hline
\end{tabular}

Values are presented as number (\%) or median (interquartile range), unless otherwise indicated. RP, radical prostatectomy; $\mathrm{RT} \pm \mathrm{ADT}$, radiotherapy with or without androgen deprivation therapy; $\mathrm{BCR}$, biochemical recurrence; BCRFS, BCR-free survival; CRPC, castration-resistant prostate cancer. 


\section{Statistical analysis}

The chi-square test and ANOVA were used to compare two or more variables, and the Mann-Whitney U test was used for the analysis of continuous variables. To address imbalances in the distribution of covariates among treatment groups, we calculated propensity scores for each subject by using multivariable logistic regression based on patient age, preoperative PSA, biopsy Gleason score, clinical tumor stage, and Charlson Comorbidity Index (CCI). Fine and Gray competing risk regression analysis was used to evaluate the association of clinical covariates with CSM and OCM. Survival endpoints were estimated and compared using the Kaplan-Meier method and a log-rank test. Statistical analyses were performed using R ver. 3.0.1 (R Foundation for Statistical Computing, Vienna, Austria). All tests were two-sided, with a statistical significance set at $p<0.05$.

Table 4. Pretreatment predictors of cancer-specific mortality in patients with clinically localized or locally advanced prostate cancer

\begin{tabular}{|c|c|c|c|c|}
\hline \multirow{2}{*}{ Variable } & \multicolumn{2}{|c|}{ Univariate } & \multicolumn{2}{|c|}{ Multivariate } \\
\hline & $\operatorname{HR}(95 \% \mathrm{CI})$ & p-value & $\mathrm{HR}(95 \% \mathrm{CI})$ & p-value \\
\hline \multicolumn{5}{|l|}{ Age (yr) } \\
\hline$<70$ & 1 (reference) & & - & \\
\hline$\geq 70$ & $0.288(0.060-1.391)$ & 0.121 & - & - \\
\hline Body mass index & $0.953(0.753-1.206)$ & 0.687 & - & - \\
\hline Pretreatment PSA & $1.012(0.973-1.054)$ & 0.545 & - & - \\
\hline \multicolumn{5}{|c|}{ Biopsy Gleason score } \\
\hline$\leq 7$ & 1 (reference) & & 1 (reference) & \\
\hline$\geq 8$ & $7.974(1.649-38.56)$ & 0.010 & $8.107(1.676-39.21)$ & 0.009 \\
\hline \multicolumn{5}{|l|}{ Clinical T stage } \\
\hline$\leq \mathrm{T} 2$ & 1 (reference) & & - & \\
\hline$\geq \mathrm{T} 3$ & $1.311(0.323-5.316)$ & 0.704 & - & - \\
\hline \multicolumn{5}{|l|}{ CCI } \\
\hline$\leq 1$ & 1 (reference) & & - & \\
\hline$\geq 2$ & $2.688(0.719-10.05)$ & 0.142 & - & - \\
\hline \multicolumn{5}{|c|}{ Initial treatment modality } \\
\hline $\mathrm{RP}$ & 1 (reference) & & - & \\
\hline $\mathrm{RT} \pm \mathrm{ADT}$ & $0.686(0.182-2.588)$ & 0.578 & - & - \\
\hline
\end{tabular}

HR, hazard ratio; CI, confidence interval; PSA, prostate-specific antigen; CCI, Charlson comorbidity index; RP, radical prostatectomy; $\mathrm{RT} \pm \mathrm{ADT}$, radiotherapy with or without androgen deprivation therapy.

\section{Patient characteristics}

Propensity matching yielded $339 \mathrm{RT} \pm \mathrm{ADT}$ cases matched to an equal number of RP cases. Clinicopathological characteristics of the two groups for matched and unmatched variables are presented in Table 1 . Variables used for propensityscore matching did not differ significantly between the two groups; this finding was confirmed by the comparable distribution of the NCCN risk criteria subgroups between the two groups. The median follow-up period of the overall cohort was 65.0 months (IQR, 40.0 to 95.0 months), with no significant differences between the two treatment groups $(\mathrm{p}=0.789)$.

\section{Causes of death}

The causes of death according to treatment modality are presented in Table 2. The causes of death were attributed to PCa and other causes in 10/678 (1.5\%) and 65/678 (9.6\%) patients, respectively. The overall mortality $(\mathrm{OM})$ rate in the $\mathrm{RT} \pm \mathrm{ADT}$ group was significantly higher than that in the RP group $(13.6 \%$ vs. $8.6 \%, p=0.038)$ because of a higher rate of OCM in the RT \pm ADT group ( $\mathrm{p}=0.013)$. The most common

\section{Results}


Table 5. Pretreatment predictors of other-cause mortality in patients with clinically localized or locally advanced prostate cancer

\begin{tabular}{|c|c|c|c|c|}
\hline \multirow{2}{*}{ Variable } & \multicolumn{2}{|c|}{ Univariate } & \multicolumn{2}{|c|}{ Multivariate } \\
\hline & HR $(95 \% \mathrm{CI})$ & p-value & HR $(95 \%$ CI $)$ & p-value \\
\hline \multicolumn{5}{|l|}{ Age (yr) } \\
\hline$<70$ & 1 (reference) & & 1 (reference) & \\
\hline$\geq 70$ & $1.684(0.992-2.860)$ & 0.053 & $1.894(1.105-3.249)$ & 0.020 \\
\hline Body mass index & $0.895(0.816-0.982)$ & 0.019 & $0.924(0.842-1.013)$ & 0.091 \\
\hline Pretreatment PSA & $1.010(0.995-1.025)$ & 0.207 & - & - \\
\hline \multicolumn{5}{|c|}{ Biopsy Gleason score } \\
\hline$\leq 7$ & 1 (reference) & & - & \\
\hline$\geq 8$ & $0.976(0.572-1.665)$ & 0.929 & - & - \\
\hline \multicolumn{5}{|l|}{ Clinical $\mathrm{T}$ stage } \\
\hline$\leq \mathrm{T} 2$ & 1 (reference) & & - & \\
\hline$\geq \mathrm{T} 3$ & $1.222(0.718-2.078)$ & 0.460 & - & - \\
\hline \multicolumn{5}{|l|}{ CCI } \\
\hline$\leq 1$ & 1 (reference) & & 1 (reference) & \\
\hline$\geq 2$ & $2.837(1.532-5.252)$ & 0.001 & $2.853(1.536-5.301)$ & 0.001 \\
\hline \multicolumn{5}{|c|}{ Initial treatment modality } \\
\hline $\mathrm{RP}$ & 1 (reference) & & 1 (reference) & \\
\hline $\mathrm{RT} \pm \mathrm{ADT}$ & $1.684(0.992-2.860)$ & 0.053 & $1.672(0.978-2.858)$ & 0.061 \\
\hline
\end{tabular}

$\mathrm{HR}$, hazard ratio; $\mathrm{CI}$, confidence interval; PSA, prostate-specific antigen; $\mathrm{CCI}$, Charlson comorbidity index; RP, radical prostatectomy; RT \pm ADT, radiotherapy with or without androgen deprivation therapy.

cause of OCM was second primary malignancy, followed by cardiopulmonary disease.

\section{Survival outcome}

Cumulative incidence estimates of CSM and OCM according to treatment modality are presented in Table 2 . Both treatments resulted in comparable CSM in all NCCN risk groups $(\mathrm{p}=0.576)$. However, the OCM rate in the $\mathrm{RT} \pm \mathrm{ADT}$ group was marginally higher than that in the $\mathrm{RP}$ group $(\mathrm{p}=0.051)$. This could be attributed to a significantly higher rate of OCM in high-risk patients who received $\mathrm{RT} \pm \mathrm{ADT}(\mathrm{p}=0.011)$. Accordingly, a higher rate of $\mathrm{OM}$ was observed in high-risk patients in the RT \pm ADT group ( $\mathrm{p}=0.047$ ).

Outcomes of secondary study endpoints are described in Table 3. Patients who received RT \pm ADT had higher BCRFS rates than those who underwent $R P(p<0.001)$. However, this did not translate into superior oncological outcomes in terms of rates of adjuvant therapy, CRPC progression-free survival, or metastasis-free survival.

\section{Predictors of cancer-specific mortality and OCM}

A multivariate competing risks regression model was used to identify pretreatment predictors of CSM and OCM. Gleason score $\geq 8$ was significantly associated with a higher risk of CSM (Table 4). Age $\geq 75$ years and CCI $\geq 2$ were independent predictors of a higher risk of OCM (Table 5).

\section{Discussion}

Our results compare favorably with those of two randomized trials reported in the literature, which compared survival outcomes of RP with those of RT $[17,18]$. Akakura et al. [17] reported no significant differences in CSM or overall survival according to treatment modality in patients with T2b3N0M0 PCa. The recently published ProtecT study also revealed no difference in CSM between the RP and RT groups [18]. However, the limitation of this study was that men who received RP were younger and had lower PSA compared to the RT group. Moreover, CCI was not accounted for. In contrast, several observational studies have reported that OM, CSM, and/or metastatic progression associated with RP are better than those associated with RT, which contradicts the findings of the present study [5-8, 19,20]. Albertsen et al. [7] reported that the CSM associated with RP was lower than that associated with RT during a 
13-year follow-up. Although PCa risk and comorbidity were adjusted for, their study did not reflect the current standard of care because it was conducted during the early PSA era [7]. Tewari et al. [19] used propensity risk scoring and reported that CSM and OM in patients with high grade $\mathrm{PCa}$ treated with RP were lower than those in patients treated with EBRT. Zelefsky et al. [6] and Merglen et al. [5] reported that CSM in men treated with RT was higher than that in men who underwent RP. However, patients treated with RT tended to be older, with higher PSA and Gleason scores, precluding a meaningful comparison. Furthermore, no adjustments were made for comorbidity $[5,6]$. The superiority of $\mathrm{RP}$ observed in these studies may be attributed to the improved ability to interpret early post-treatment PSA changes and to deliver timely and effective adjuvant therapy by enabling a pathologic assessment of the primary tumor [20]. Moreover, patients for whom RP was deemed appropriate may have been better screened for second primary malignancies or comorbidities such as cardiopulmonary disease, which contributes to OCM. Nevertheless, the most reliable quantitative exploratory analyses, including the aforementioned observational studies, concluded that the differences in 10-year CSM are less than 1\%, and that the unadjusted survival curves and unaccounted-for confounders in these studies preclude a definitive conclusion that RP results in superior survival compared to RT \pm ADT [21].

Patients treated with $\mathrm{RT} \pm \mathrm{ADT}$ exhibited higher BCRFS rates than those who underwent RP. However, this finding did not translate into improvement in consequent oncological outcomes during our observational period. The high rate of ADT administration in our patients treated with RT may have contributed to this result. Overall, $78 \%$ of the patients who received RT in the current analysis also received neoadjuvant, concomitant, and/or adjuvant ADT. This proportion is markedly higher than the $51 \%$ to $56 \%$ reported in previous studies [6]. This may be, in part, due to the higher proportion of high-risk patients in our cohort who received ADT, a practice based on evidence that RT with ADT results in better survival than that for RT alone [2]. Indeed, the use of ADT in the RT group is a potential confounding factor for comparisons between RP and RT. Nevertheless, we did not adjust for the use of ADT in the present study for several reasons. First, the use of ADT is associated with disease risk, such that higher risk patients are more likely to receive ADT. The impact of ADT is usually reflected in the risk adjustment. In previous studies using models adjusted for risk, ADT was not proven to be an independent predictor $[13,22]$. Second, BCRFS which may be affected by the use of ADT, was not the primary endpoint of our study. Biochemical recurrence is known to antedate clinical progression by a median of 5 to 7 years [23]. However, considering the protracted natural history of $\mathrm{PCa}$, biochemical recurrence is an imprecise proxy for

\section{CSM or OM.}

A noteworthy finding in the present study was that the OCM rate in patients who received $\mathrm{RT} \pm \mathrm{ADT}$ was higher than that in patients who underwent RP. Although propensityscore matching was utilized to adjust for confounding comorbidities that might have increased OCM, unobserved and unaccounted disparities between the groups may have existed. CCI has been suggested to predict the risk of OCM unreliably, and adequate adjustments are best applied when populations are more homogeneous, such as those with a CCI of $0[8,21]$. To account for this issue, we compared survival outcomes within patients with a CCI of 0 . Interestingly, no differences in OCM were observed within this subgroup (data not shown). This observation implies that CCI may fail to ensure adequate adjustments for OCM in patients with at least one or more significant comorbidity. The administration of $\mathrm{ADT}$ is another potential risk factor contributing to cardiopulmonary disease as the cause of OCM. Studies have suggested increased risk of cardiopulmonary disease with long term ADT $[24,25]$. However at the same time, there equal levels of evidences that oppose this observation [26,27]. The jury is still out whether lower levels of testosterone has caused more cardiopulmonary disease and have contributed to higher OCM rates in our patients who received RT+ADT.

The present study has a few noteworthy limitations. (1) Although we utilized a propensity-score matched analysis, unobserved and unaccounted disparities between cohorts may have existed, as evidenced by our subset analysis of patients without any comorbidities. (2) The aim of our study was to provide a guide to aid clinical decision making at diagnosis. Thus, no adjustments were made for confounders that may contribute to survival following initial treatment, namely, salvage therapy, duration of ADT, or administration of chemotherapy or androgen receptor-targeted therapy. Although studies comparing RP and RT have reported that adjustment for salvage therapy had no impact on survival outcomes [6], it is difficult to preclude the effect of salvage therapy considering multidisciplinary treatment strategies and the prolonged natural history of PCa. (3) Treatments are constantly evolving and advances in radiation delivery may have some impact of survival. Randomized trials have demonstrated dose-escalated RTx (74-90 Gy) to improve BCRFS compared to conventional RTx (64-70 Gy) [28]. However, we did not account for this confounder because none have demonstrated improvement in CSM or OM with higher doses or variations in technique, such as intensity modulation $[29,30]$. Moreover, no differences in BCRFS or CSM were noted according to radiation dose subgroups (data not shown). (4) A limited number of cases of CSM occurred in the low-risk group, which limited our ability to draw statistically significant conclusions. (5) The follow-up period was relatively short compared to previous similar studies. 
Although higher BCRFS rates observed in patients treated with $\mathrm{RT} \pm \mathrm{ADT}$ did not translate into superior oncological endpoints, a longer observational period would be needed for a meaningful comparison of overall survival. (6) We did not investigate the differences in adverse events and quality of life, which are meaningful clinical endpoints.

$\mathrm{RP}$ and RT $\pm \mathrm{ADT}$ yield comparable CSM outcomes in Korean patients with localized or locally advanced PCa. Our results imply that the risk of OCM may be higher for older high-risk patients with significant comorbidities. Future investigations focusing on long-term cancer control as well as functional and patient satisfaction outcomes will be necessary for a more definitive conclusion.

\section{Conflicts of Interest}

Conflict of interest relevant to this article was not reported.

\section{Acknowledgments}

This study was supported by a faculty research grant of Yonsei University College of Medicine (6-2016-0105).

\section{References}

1. Heidenreich A, Bastian PJ, Bellmunt J, Bolla M, Joniau S, van der Kwast T, et al. EAU guidelines on prostate cancer. part 1: screening, diagnosis, and local treatment with curative intentupdate 2013. Eur Urol. 2014;65:124-37.

2. Mohler JL, Armstrong AJ, Bahnson RR, D'Amico AV, Davis BJ, Eastham JA, et al. NCCN clinical practice guidelines in oncology: prostate cancer, version 3 [Internet]. Fort Washington, PA: National Comprehensive Cancer Network; 2016 [cited 2016 Mar 10]. Available from: http://www.jnccn.org/ content/8/2/162.full.pdf+html.

3. Paulson DF, Lin GH, Hinshaw W, Stephani S. Radical surgery versus radiotherapy for adenocarcinoma of the prostate. J Urol. 1982;128:502-4.

4. Wallace K, Fleshner N, Jewett M, Basiuk J, Crook J. Impact of a multi-disciplinary patient education session on accrual to a difficult clinical trial: the Toronto experience with the surgical prostatectomy versus interstitial radiation intervention trial. J Clin Oncol. 2006;24:4158-62.

5. Merglen A, Schmidlin F, Fioretta G, Verkooijen HM, Rapiti E, Zanetti R, et al. Short- and long-term mortality with localized prostate cancer. Arch Intern Med. 2007;167:1944-50.

6. Zelefsky MJ, Eastham JA, Cronin AM, Fuks Z, Zhang Z, Yamada $Y$, et al. Metastasis after radical prostatectomy or external beam radiotherapy for patients with clinically localized prostate cancer: a comparison of clinical cohorts adjusted for case mix. J Clin Oncol. 2010;28:1508-13.

7. Albertsen PC, Hanley JA, Penson DF, Barrows G, Fine J. 13year outcomes following treatment for clinically localized prostate cancer in a population based cohort. J Urol. 2007;177: 932-6.

8. Nepple KG, Stephenson AJ, Kallogjeri D, Michalski J, Grubb RL 3rd, Strope SA, et al. Mortality after prostate cancer treatment with radical prostatectomy, external-beam radiation therapy, or brachytherapy in men without comorbidity. Eur Urol. 2013;64:372-8.

9. Kibel AS, Ciezki JP, Klein EA, Reddy CA, Lubahn JD, HaslagMinoff J, et al. Survival among men with clinically localized prostate cancer treated with radical prostatectomy or radiation therapy in the prostate specific antigen era. J Urol. 2012;187: 1259-65.

10. Sooriakumaran P, Nyberg T, Akre O, Haendler L, Heus I, Olsson $\mathrm{M}$, et al. Comparative effectiveness of radical prostatectomy and radiotherapy in prostate cancer: observational study of mortality outcomes. BMJ. 2014;348:g1502.

11. Concato J, Shah N, Horwitz RI. Randomized, controlled trials, observational studies, and the hierarchy of research designs. N Engl J Med. 2000;342:1887-92.

12. Donovan JL, Lane JA, Peters TJ, Brindle L, Salter E, Gillatt D, et al. Development of a complex intervention improved randomization and informed consent in a randomized controlled trial. J Clin Epidemiol. 2009;62:29-36.

13. Cooperberg MR, Vickers AJ, Broering JM, Carroll PR. Comparative risk-adjusted mortality outcomes after primary surgery, radiotherapy, or androgen-deprivation therapy for localized prostate cancer. Cancer. 2010;116:5226-34.

14. Penson DF. An update on randomized clinical trials in localized and locoregional prostate cancer. Urol Oncol. 2005;23: 280-8.

15. Rubin DB. On principles for modeling propensity scores in medical research. Pharmacoepidemiol Drug Saf. 2004;13: 855-7.

16. Boehmer D, Maingon P, Poortmans P, Baron MH, Miralbell R, Remouchamps $\mathrm{V}$, et al. Guidelines for primary radiotherapy of patients with prostate cancer. Radiother Oncol. 2006;79: 259-69.

17. Akakura K, Suzuki H, Ichikawa T, Fujimoto H, Maeda O, Usami $\mathrm{M}$, et al. A randomized trial comparing radical prostatectomy plus endocrine therapy versus external beam radiotherapy plus endocrine therapy for locally advanced prostate cancer: results at median follow-up of 102 months. Jpn J Clin Oncol. 2006;36:789-93.

18. Johnston TJ, Shaw GL, Lamb AD, Parashar D, Greenberg D, Xiong $\mathrm{T}$, et al. Mortality among men with advanced prostate cancer excluded from the ProtecT Trial. Eur Urol. 2017;71: 
381-8.

19. Tewari A, Divine G, Chang P, Shemtov MM, Milowsky M, Nanus D, et al. Long-term survival in men with high grade prostate cancer: a comparison between conservative treatment, radiation therapy and radical prostatectomy: a propensity scoring approach. J Urol. 2007;177:911-5.

20. Lee BH, Kibel AS, Ciezki JP, Klein EA, Reddy CA, Yu C, et al. Are biochemical recurrence outcomes similar after radical prostatectomy and radiation therapy? Analysis of prostate cancer-specific mortality by nomogram-predicted risks of biochemical recurrence. Eur Urol. 2015;67:204-9.

21. Roach M 3rd, Ceron Lizarraga TL, Lazar AA. Radical prostatectomy versus radiation and androgen deprivation therapy for clinically localized prostate cancer: how good is the evidence? Int J Radiat Oncol Biol Phys. 2015;93:1064-70.

22. Cooperberg MR, Grossfeld GD, Lubeck DP, Carroll PR. National practice patterns and time trends in androgen ablation for localized prostate cancer. J Natl Cancer Inst. 2003;95: 981-9.

23. Stephenson AJ, Kattan MW, Eastham JA, Dotan ZA, Bianco FJ Jr, Lilja $\mathrm{H}$, et al. Defining biochemical recurrence of prostate cancer after radical prostatectomy: a proposal for a standardized definition. J Clin Oncol. 2006;24:3973-8.

24. D'Amico AV, Denham JW, Crook J, Chen MH, Goldhaber SZ, Lamb DS, et al. Influence of androgen suppression therapy for prostate cancer on the frequency and timing of fatal myocardial infarctions. J Clin Oncol. 2007;25:2420-5.

25. Keating NL, O'Malley AJ, Freedland SJ, Smith MR. Diabetes and cardiovascular disease during androgen deprivation therapy: observational study of veterans with prostate cancer. J Natl Cancer Inst. 2010;102:39-46.

26. Nguyen PL, Je Y, Schutz FA, Hoffman KE, Hu JC, Parekh A, et al. Association of androgen deprivation therapy with cardiovascular death in patients with prostate cancer: a metaanalysis of randomized trials. JAMA. 2011;306:2359-66.

27. Efstathiou JA, Bae K, Shipley WU, Hanks GE, Pilepich MV, Sandler HM, et al. Cardiovascular mortality and duration of androgen deprivation for locally advanced prostate cancer: analysis of RTOG 92-02. Eur Urol. 2008;54:816-23.

28. Moon DH, Efstathiou JA, Chen RC. What is the best way to radiate the prostate in 2016? Urol Oncol. 2017;35:59-68.

29. Wilt TJ, MacDonald R, Rutks I, Shamliyan TA, Taylor BC, Kane RL. Systematic review: comparative effectiveness and harms of treatments for clinically localized prostate cancer. Ann Intern Med. 2008;148:435-48.

30. Zietman AL, DeSilvio ML, Slater JD, Rossi CJ Jr, Miller DW, Adams JA, et al. Comparison of conventional-dose vs highdose conformal radiation therapy in clinically localized adenocarcinoma of the prostate: a randomized controlled trial. JAMA. 2005;294:1233-9. 